\title{
GPS-Based Daily Context Recognition for Lifelog Generation Using Smartphone
}

\author{
Go Tanaka \\ Graduate School of Informatics, \\ Shizuoka University \\ Hamamatsu, Japan
}

\author{
Masaya Okada \\ Graduate School of Informatics, \\ Shizuoka University \\ Hamamatsu, Japan
}

\author{
Hiroshi Mineno \\ Graduate School of Informatics, \\ Shizuoka University \\ Hamamatsu, Japan
}

\begin{abstract}
Mobile devices are becoming increasingly more sophisticated with their many diverse and powerful sensors, such as GPS, acceleration, and gyroscope sensors. They provide numerous services for supporting daily human life and are now being studied as a tool to reduce the worldwide increase of lifestyle-related diseases. This paper describes a method for recognizing the contexts of daily human life by recording a lifelog based on a person's location. The proposed method can distinguish and recognize several contexts at the same location by extracting features from the GPS data transmitted from smartphones. The GPS data are then used to generate classification models by machine learning. Five classification models were generated: a mobile or stationary recognition model, a transportation recognition model, and three daily context recognition models. In addition, optimal learning algorithms for machine learning were determined. The experimental results show that this method is highly accurate. As examples, the Fmeasure of the daily context recognition was approximately 0.954 overall at a tavern and approximately 0.920 overall at a university ${ }^{1}$.
\end{abstract}

Keywords-component; Lifelog; machine learning; GPS; healthcare

\section{INTRODUCTION}

The use of mobile devices such as smartphones and tablets has become more widespread and sophisticated. These devices contain many diverse and powerful sensors, such as GPS sensors, acceleration sensors, and gyroscope sensors. Since the sensors are very small and lightweight, they can collect various types of personal data without inconveniencing the user. Mining the collected data helps us to learn many details about the daily lives of people. The knowledge gained from data mining can be applied to various individual life support services such as healthcare services and online-to-offline (O2O) services. Individuals can also use the knowledge obtained from a lifelog recorded by the model devices. The lifelog, which captures all or a large portion of one's life, can be referenced to learn about one's own experiences and lifestyle. Services related to lifelogs have recently been attracting attention [1]-[3]. High-quality lifelogs can speed up

\footnotetext{
${ }^{1}$ G. Tanaka is with the Graduate School of Informatics, Shizuoka University, Japan

M. Okada is with the Graduate School of Informatics, Shizuoka University, Japan

H. Mineno is with the Graduate School of Informatics, concurrently with the Graduate School of Science and Technology, the Research Institute of Green Science and Technology, Shizuoka University, Japan
}

the development of essential services, especially in healthcare. This is an important goal due to the rapid increase in the number of people who die from lifestyle-related diseases such as cancer, heart disease, and strokes. The World Health Organization (WHO) refers to lifestyle-related diseases as noncommunicable diseases (NCDs) and states that 36 million people, or $63 \%$ of the 57 million global deaths, died from NCDs in 2008 [4]. The WHO further estimates that the total number of annual NCD deaths is projected to reach 55 million by 2030. In Japan, NCDs have become the primary cause of death. Therefore, people should examine their own daily lives to improve their lifestyles.

In an extension of a previous study [5], this paper proposes a method for daily context recognition. This method generates high-quality lifelogs by using only a GPS sensor. Because locations and activities are important elements for a daily lifelog, this method recognizes the user's location and activity as contexts. It also helps to distinguish and recognize several contexts that are considered to appear at the same location.

The proposed method measures several variables captured by a commercial mobile phone for daily context recognition of a person's activity. The user's activities captured by the mobile phone show their habits over time. Therefore, the user can look back at his or her daily life in more detail by routinely carrying a smartphone. In addition, it becomes possible to create and provide services in accordance with the user's location and situation. The intended result is an improved lifestyle for the prevention of lifestyle-related diseases.

\section{RELATED WORK}

Lifelog generation using human activity recognition technologies has recently gained attention as a research topic. Research on healthcare for lifestyle-related diseases has also been increasing. Much of the research focuses on monitoring and recognizing human activity to give feedback to the user. Results of some of the research on human activity recognition and healthcare related to lifelogs are described below.

\section{A. Human Activity Recognition}

In one of the earliest studies on human activity recognition, Lara and Labrador [6] used three sensing devices-GPS, accelerometers, and vital signs - and created decision tree models to recognize three basic physical activities-walking, running, and sitting - by using the $\mathrm{C} 4.5$ classification algorithm. Gomes et al. [7] developed a mobile activity recognition system (MARS) that learns the classification model onboard 
the mobile device itself through ubiquitous data stream mining in an incremental manner. Using the naive Bayes classification algorithm along with acceleration data, they created personal models to recognize five physical activities - walking, running, standing still, driving, and climbing stairs. Kwapisz et al. [8] used accelerometers to recognize daily physical activities. They compared three classification algorithms - the J48 decision tree, logistic regression, and the multilayer perceptron - and found that the multilayer perceptron performed the best overall for recognizing six physical activities and actions (walking, jogging, climbing stairs, going down stairs, sitting, and standing). Hattori et al. [9] developed the ALKAN system. ALKAN is a server-client system that gathers a large number of "missions" by using mobile sensor devices with accelerometers. They recognized eight physical activitieseating, cycling, riding in a car, sitting, standing, sitting in a train, standing in a train, and walking. For machine learning training, they used four classification algorithms: the recursive partitioning tree, naive Bayes classifier, nearest neighbor classification, and support vector machine (SVM). Many other researchers have also used machine learning for human activity recognition, and some of them have used accelerometer data [10]-[18].

All of the above studies recognize only basic human physical activities or attitudes; none recognize the purpose for an activity in a person's daily life. Therefore, these methods do not obtain the information for generating lifelogs, and users cannot look back at the contexts of their daily lives to improve their lifestyles. Furthermore, some problems must be considered when using acceleration sensors. First, the accuracy of the recognition when using acceleration sensors depends on the mounting position of the sensor device. The mounting position of wearable sensors is especially important when the user's mobile phone contains an accelerometer. Second, it is impossible to gather accurate acceleration sensing data when the user is actively using his or her phone, because too much noise is generated while touching the smartphone.

The method presented in this paper is a novel method that can gather accurate sensing data regardless of the wearable position and operation, and can accurately recognize specific contexts of daily life.

\section{B. Healthcare}

In the healthcare research field, many participants use wearable sensor devices or smartphones with acceleration sensors, GPS, and so on, for monitoring and managing the user's lifestyle and personal medical information [19]-[29]. Suzuki et al. [19] proposed improving lifestyles from the perspective of a user's meal choice. They analyzed the user's situation, biological information, and lifestyle (e.g., budget, workload, and spare time) and then provided advice on purchasing appropriate foods. Yan et al. [20] used body sensors for monitoring the movement of elderly people. They proposed a mixed positioning algorithm to determine the location of an elderly person to determine that person's activities and to make decisions about his/her health status. Khan et al. [21] used an accelerometer in a smartphone to recognize a person's daily physical activities in order to suggest minor behavioral modifications to increase the energy expenditure in one's daily routine.

Although most of the above studies use a wearable device, the focus is almost entirely on treating diseases and health problems, and there is little focus on actually preventing them. According to the WHO, the highest probability of dying from an NCD is between the ages of 30 and 70 in many locations around the globe, and the accrual of improper lifestyles from a young age can be thought of as one of the reasons for a later NCD; that is, by the time a person is old, it may be too late. Therefore, it is necessary for people to monitor and improve their own daily lifestyles from a young age to prevent later lifestyle-related diseases.

\section{DAILY CONTEXT RECOGNITION}

The method proposed in this paper has the following requirements:

1) Recognizing not only basic physical activities but also specific contexts related to daily life.

2) Gathering accurate data regardless of the wearable position and operation of the sensor device.

3) Reducing the installation cost so that young people can use it.

4) Preventing lifestyle-related diseases by improving the user's lifestyle.

The proposed method estimates the user's location and his or her contexts at any given moment. For example, several contexts can occur at one given location, such as a shopping mall. This method can distinguish between shopping, eating a meal, and seeing a movie at the shopping mall. Daily activities (with the exception of home activities, which were the focus of a previous paper [30]) are estimated in this paper.

The contexts for lifelog generation are estimated by data mining using sensor data. This paper uses a GPS sensor in a smartphone because such sensor data do not depend on the user's position or operation. Moreover, because individual differences rarely appear, it is possible to generate a generic model. The user needs only a smartphone, and so the installation cost is low. All of this is intended to generate lifelogs for the prevention of lifestyle-related diseases.

Figure 1 shows a flowchart for recognizing daily contexts. Most recognition processors use machine learning. The Weka [31] data mining software was used for the machine learning in this work. Details of the proposed method are described in the following.

\section{A. Feature Extraction}

The feature extraction processor extracts five features for machine learning: speed, variance, weather, time zone, and day of the week. First, the GPS obtains the latitude, longitude, and time. The speed is calculated on the basis of the distance between two location points, which are determined by latitude (lat) and longitude (lon) values, and then this value is divided by the difference between the times of data collection. The variance of location is calculated in (1). In this paper, the number of longitudinal data $n$ is 5 . 


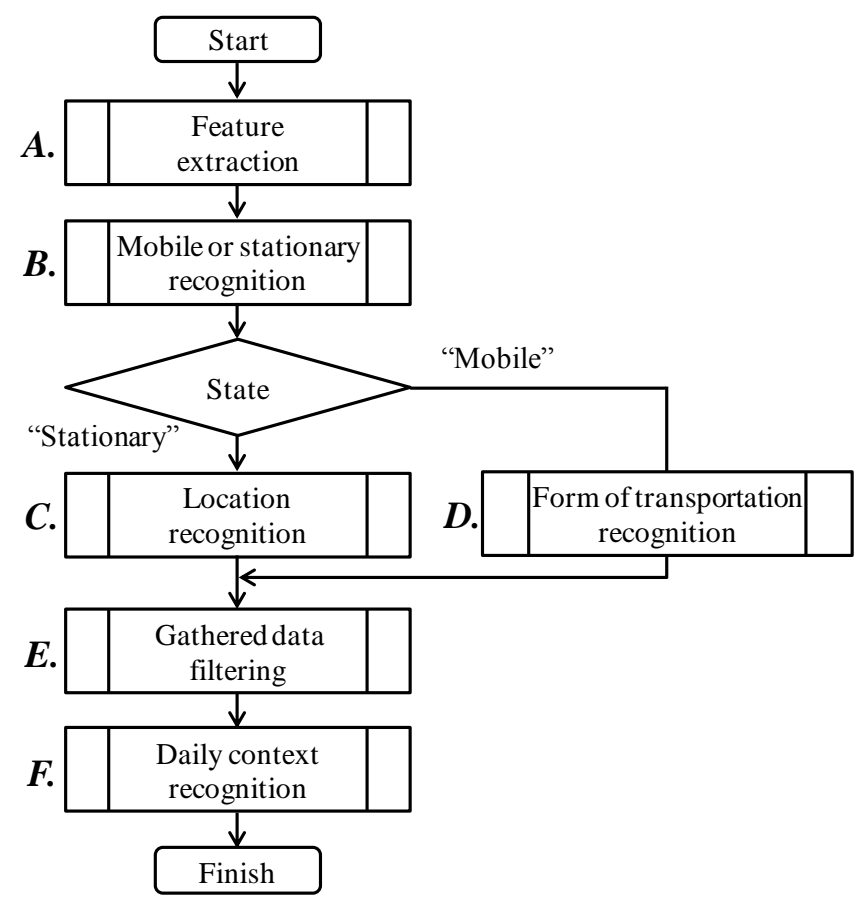

Fig. 1. Flowchart of user's daily context recognition using only a GPS sensor

$$
\sqrt{\left\{\frac{1}{n} \sum_{i=1}^{n}\left(\overline{\text { lat }}-\text { lat }_{i}\right)^{2}\right\}^{2}+\left\{\frac{1}{n} \sum_{i=1}^{n}\left(\overline{\text { lon }}-\text { lon }_{i}\right)^{2}\right\}^{2}}
$$

Weather is obtained from a Meteorological Agency site according to the given time. The time zone is defined as late_night (0:00 to 2:00), early_morning $(2: 00$ to $6: 00)$, forenoon (6:00 to 10:00), noon (10:00 to 14:00), afternoon (14:00 to $18: 00)$, night (18:00 to $22: 00)$, and late_night (22:00 to $24: 00)$.

\section{B. Mobile or Stationary Recognition}

The next step is to recognize whether a user is mobile or stationary in order to recognize their location. The mobile or stationary condition needs to be simply and very accurately recognized because this process is the first step in recognizing daily contexts. Machine learning is used for recognizing whether the user is mobile or stationary. The explanatory variables for machine learning are the speed and the variance of the location. The user is considered to be mobile if the speed or the variance is greater, and vice versa.

\section{Location Recognition}

This processor recognizes a stationary location by recognizing whether the person is mobile. By registering the information of locations in a database well in advance, it is possible to easily estimate the user's location when they are stationary at a registered position. If the user is stationary at an unregistered position, it is possible to obtain information from nearby facilities by a place search API (a service that returns information about places by HTTP requests).

\section{Form of Transportation Recognition}

This processor recognizes the type of transportation when the person is mobile. The form of transportation is used to determine the number of non-exercise activity thermogenesis (NEAT) calories and is one of the explanatory variables in machine learning. Since NEAT includes all daily life activities, it is possible to prevent lifestyle-related diseases by promoting non-exercise daily activities. The type of transportation used is an explanatory variable because people change the form of transportation on the basis of the purpose and situation at their given location. By also using speed, location variance, and the weather as explanatory variables, it is possible to recognize five forms of transportation: on foot (Walk), riding a scooter (Scooter), driving in a car (Car), taking a train (Train), and taking the Shinkansen (Shinkansen).

\section{E. Data Filtering}

The GPS data collected by a smartphone are filtered to improve the level of accuracy for recognizing daily contexts. The positioning error is significantly worse indoors with the GPS sensor. Therefore, a lot of generated noise must be removed by filtering. However, since general filtering methods such as the moving average filter and the low-pass filter are used for smoothing longitudinal data, they cannot respond to rapid and large changes in a user's position (e.g., transition from a stopped state while on the Shinkansen). Therefore, this processor removes the noise data by using accuracy values obtained from the GPS sensor of a general OS-based mobile phone. The level of accuracy is given in meters as the error range of the distance. The accuracy is defined as a $68 \%$ confidence radius. By appropriately setting a threshold for the accuracy values, it is possible to improve the recognition accuracy by removing the noise.

\section{F. Daily Context Recognition}

Finally, this paper recognizes the contexts for the stationary location related to a person's daily life. It is possible to recognize not only basic human physical activities but also a user's location (i.e., daily location) and context (i.e., what the user is doing there) by generating context recognition models. It is possible to determine a person's poor lifestyle habits, improve them, and help to prevent lifestyle-related diseases by recognizing the contexts that account for most of his or her daily life at a specific location. The location where the contexts have been recognized-e.g., a restaurant, university, convenience store, or shopping mall-is registered in the database, and after that, the same model can be used when the user visits a similar location for the first time.

\section{EXPERIMENT}

This section describes our initial experiments to evaluate the proposed method for recognizing daily human contexts. As mentioned above, because users need to improve their lifestyles from a young age, experiments were conducted based on the daily activities of students. Figure 2 shows the daily activity model of an example student. 


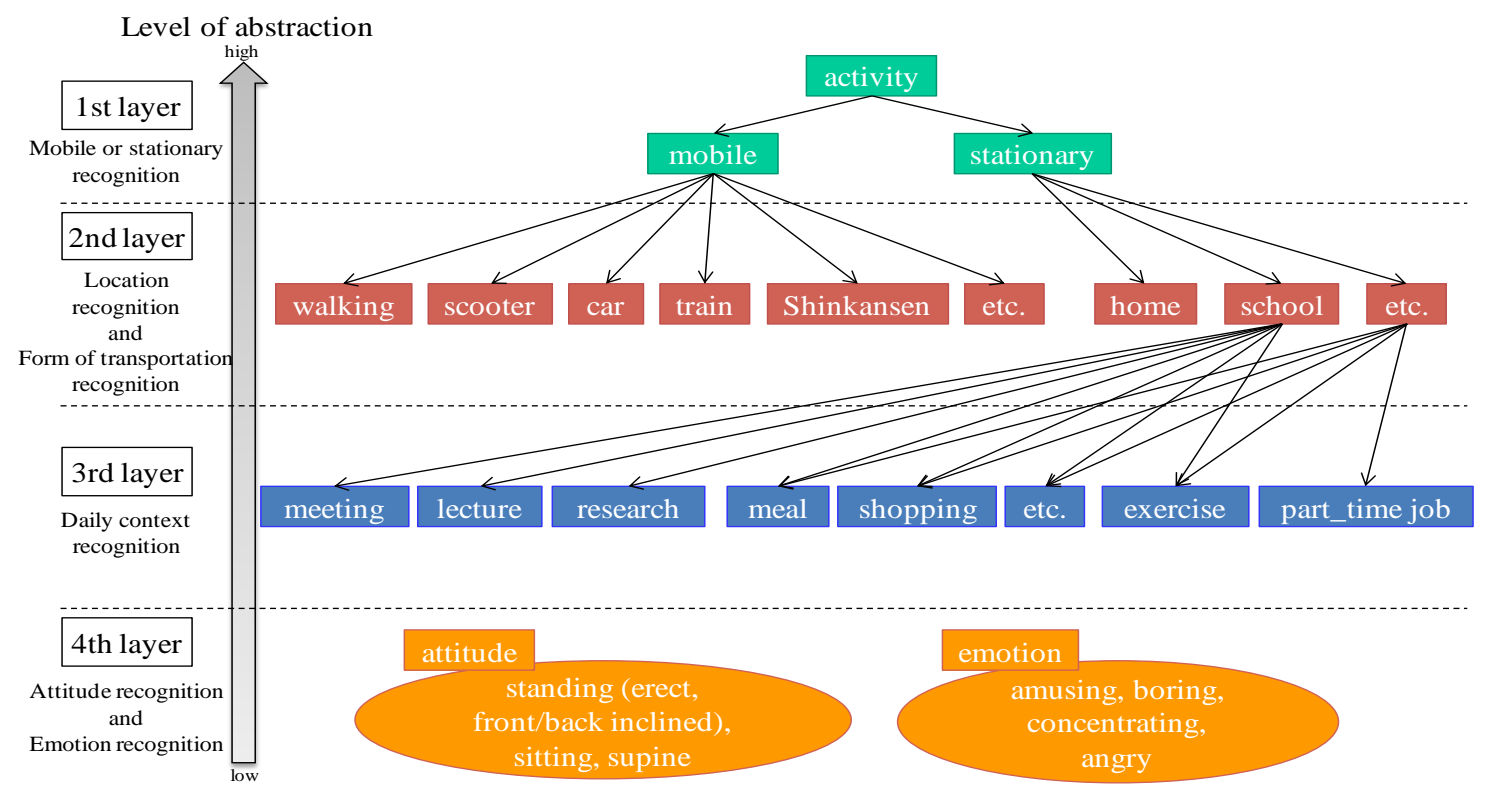

Fig. 2. Daily activity model of a student. Model is divided into four layers referring to different levels of abstraction

The activities of all participants are divided into mobile and stationary. Students walk, ride a scooter, drive a car, board a train, board the Shinkansen, and so on as primary mobile activities while their home, school, etc. are the primary places of stationary activities. Contexts such as meetings, lectures, research, meals, shopping, exercise, and part-time jobs occur at the stationary places. In addition to these activities and contexts, actions such as sitting and standing are recorded at all times. It is clear that people who walk on a regular basis are healthier than people who get into a car or ride a scooter. It is also possible to call attention to people who eat meals at irregular times or who do not eat at all. Moreover, the effect on lifestylerelated diseases depends on the user's context, even when people are stationary at the same location. For example, a student's expenditure of calories increases by working a parttime job at a restaurant, but the calorie intake increases when they eat a meal there. Therefore, it is necessary to distinguish between and recognize the contexts that indicate a difference in calorie consumption. The lifelog generated by recognizing the contexts based on this model could improve the lifestyles of students.

We investigated three details in the experiments: recognition accuracy, validity of the explanatory variables for machine learning, and the optimal machine learning algorithm. The recognition accuracy is evaluated by F-measure $F_{l}$, as

$$
F_{1}=\frac{2 \times \text { recall } \times \text { precision }}{\text { recall }+ \text { precision }}
$$

$F_{l}$ is a performance measure widely adopted in the fields of pattern recognition and information retrieval. It is the harmonic means of both recall and precision. Various combinations of explanatory variables were compared in experiments to confirm their validity. Random forest, J48 based on C4.5, SVM, neural network, and Bayesian network algorithms were compared to determine the optimal algorithm for machine learning. Default WEKA functions were employed to ensure a ten-fold cross validation in all experiments. The purpose of machine learning was classification, so we used support vector classification (SVC). Parameters such as cost and gamma were tuned by using a grid search because SVM is an algorithm that considers parameter tuning as the most important operation. The radial basis function (RBF) was used as the kernel function.

\section{A. Experimental Results}

The accuracy of mobile or stationary recognition, the form of transportation recognition, and the daily context recognition were evaluated in the initial experiments. The recognized contexts were the locations at a tavern and at a university, both of which are places assumed to be frequented by students in their daily lives.

\section{1) Mobile or stationary recognition}

GPS data from five participants were collected for two weeks. Figure 3 shows the accuracies of mobile or stationary recognition by using the random forest algorithm. In the figure, the Gini is defined as the "inequity" of a society's distribution of income, or a measure of "node impurity" in a tree-based classification. TABLE I lists the correctness of the models generated by the five learning algorithms and the accuracies when using the test data. 

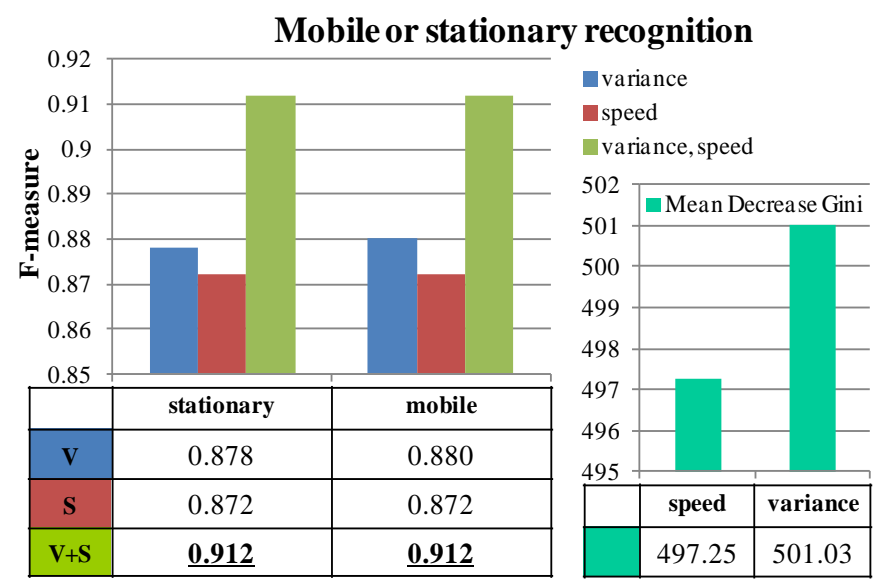

Fig. 3. Accuracies of mobile or stationary recognition by using random forest algorithm for combinations of explanatory variables, and the mean decrease Gini of explanatory variables. Highest values are in bold and underlined

TABLE I. MOdel CORRECTNESS AND ACCURACIES OF TEST DATA For MOBILE OR STATIONARY RECOGNITION

\begin{tabular}{cccccc}
\hline \hline $\begin{array}{c}\text { Model/ } \\
\text { Test }\end{array}$ & $\begin{array}{c}\text { Random } \\
\text { Forest }\end{array}$ & $\mathbf{J 4 8}$ & SVM & $\begin{array}{c}\text { Neural } \\
\text { Network }\end{array}$ & $\begin{array}{c}\text { Bayesian } \\
\text { Network }\end{array}$ \\
\hline Stationary & $0.912 /$ & $0.898 /$ & $0.890 /$ & $0.889 /$ & $0.921 /$ \\
& $\underline{\mathbf{0 . 9 2 6}}$ & 0.898 & 0.892 & 0.889 & 0.925 \\
Mobile & $0.912 /$ & $0.883 /$ & $0.879 /$ & $0.879 /$ & $0.921 /$ \\
& $\underline{\mathbf{0 . 9 2 4}}$ & 0.883 & 0.883 & 0.885 & 0.923 \\
Overall & $0.912 /$ & $0.891 /$ & $0.884 /$ & $0.884 /$ & $0.921 /$ \\
& $\mathbf{0 . 9 2 5}$ & 0.891 & 0.888 & 0.887 & 0.924 \\
\hline
\end{tabular}

Three cases were compared in the experiments: using only the location variance, using only the speed, and using both variables as explanatory variables. As shown in Figure 3, using both variables achieves the highest level of accuracy. It is also clear that the importance of both variables is significantly higher when looking at the mean decrease Gini values. A higher decrease in Gini implies that a particular predictor variable plays a greater role in partitioning the data into the defined classes. TABLE I lists the model correctness generated from a training data set of 2,000 (stationary: 1,000, mobile: $1,000)$ and the evaluation results when using the training data set and the test data set of 1,000 (stationary: 500, mobile: 500 ). As shown in this table, all cases achieve high levels of accuracy above 0.880 (F-measure). The random forest algorithm achieves the highest level of accuracy in both activities. An incorrect classification occurs when GPS data with large positioning errors from an indoor location are used in the learning and the data are recognized as "stationary" even if the speed or variance values are large.

\section{2) Form of transportation recognition}

The same GPS data collected from the same five participants for two weeks were used in this evaluation. Figure 4 shows the accuracies of the form of transportation recognition using the random forest algorithm. TABLE II lists the correctness of the models generated by the five learning algorithms and the accuracies when using the test data.

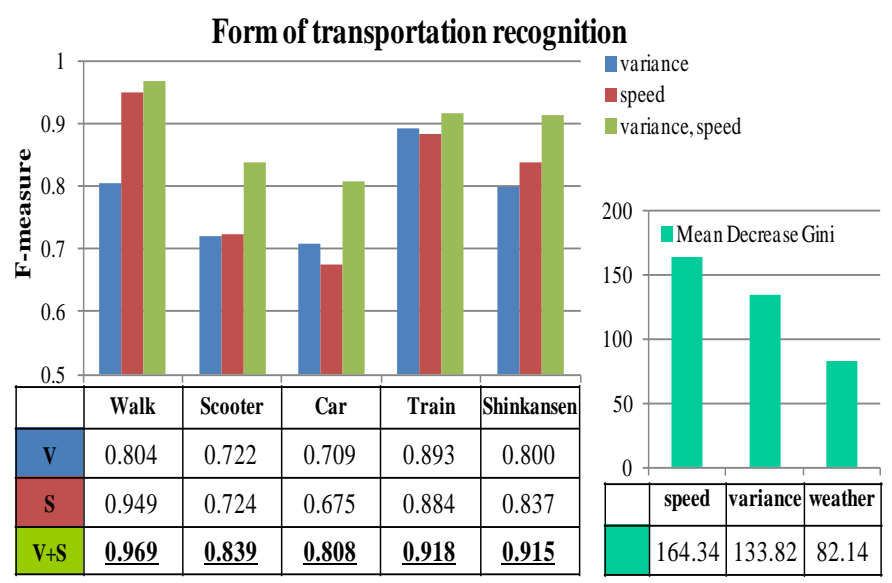

Fig. 4. Differences in accuracies of form of transportation recognition by using random forest algorithm for combinations of explanatory variables, and the mean decrease Gini of explanatory variables

TABLE II. MOdel CORReCTNESS AND ACCURACIES OF TEST DATA For FORM OF TRANSPORTATION RECOGNITION

\begin{tabular}{cccccc}
\hline \hline $\begin{array}{c}\text { Model/ } \\
\text { Test }\end{array}$ & $\begin{array}{c}\text { Random } \\
\text { Forest }\end{array}$ & $\mathbf{J 4 8}$ & SVM & $\begin{array}{c}\text { Neural } \\
\text { Network }\end{array}$ & $\begin{array}{c}\text { Bayesian } \\
\text { Network }\end{array}$ \\
\hline Walk & $0.969 /$ & $0.959 /$ & $0.962 /$ & $0.917 /$ & $0.973 /$ \\
& 0.946 & 0.951 & 0.951 & $\underline{\mathbf{0 . 9 5 9}}$ & 0.953 \\
Scooter & $0.839 /$ & $0.745 /$ & $0.657 /$ & $0.683 /$ & $0.797 /$ \\
& $\underline{\mathbf{0 . 8 6 1}}$ & 0.750 & 0.744 & 0.707 & 0.816 \\
Car & $0.808 /$ & $0.694 /$ & $0.598 /$ & $0.559 /$ & $0.653 /$ \\
& $\underline{\mathbf{0 . 8 2 8}}$ & 0.698 & 0.686 & 0.638 & 0.622 \\
Train & $\mathbf{0 . 9 1 8 /}$ & $0.881 /$ & $0.779 /$ & $0.833 /$ & $0.822 /$ \\
& $\underline{\mathbf{0 . 9 0 1}}$ & 0.855 & 0.700 & 0.754 & 0.757 \\
Shinkansen & $\mathbf{0 . 9 1 5 /}$ & $0.919 /$ & $0.779 /$ & $0.858 /$ & $0.877 /$ \\
Overall & $\underline{\mathbf{0 . 9 2 9}}$ & 0.893 & 0.813 & 0.863 & 0.863 \\
& $\mathbf{0 . 8 9 0 /}$ & $0.840 /$ & $0.759 /$ & $0.770 /$ & $0.824 /$ \\
& $\underline{\mathbf{0 . 8 9 3}}$ & 0.829 & 0.779 & 0.784 & 0.802 \\
\hline
\end{tabular}

Comparative experiments were performed in cases similar to the experiment for mobile or stationary recognition. However, weather was added to the explanatory variables in all cases because it could affect the user's behavior. As shown in Figure 4, the use of all variables ensures the highest level of accuracy. The mean decrease Gini values show that speed was the most important element for the form of transportation recognition. TABLE II lists the model correctness generated from a training data set of 750 (150 pieces of data per context) and the evaluation results when using the training data set and a test data set of 375 (75 pieces of data per context). As shown in this table, almost all cases are highly accurate, indicating that it is possible to achieve high levels of accuracy in all activities by using the random forest algorithm. The random forest algorithm achieves higher levels of accuracy than the other algorithms for Scooter, Car, and Train because it can generate a strong classifier by group learning of the decision trees. The reason for the lower levels of accuracy for Scooter and Car is assumed to be the frequent changes in car speed stemming from various traffic conditions, such as signal changes and pedestrians, and thus the car speed is often equal to the scooter speed. Therefore, Car is erroneously recognized as Scooter. 


\section{3) Daily context recognition at a tavern}

GPS data were collected for one participant (one of the authors) for one month. Figure 5 shows the accuracies of the daily context recognition at a tavern by using the J48 algorithm. TABLE III lists the model correctness generated by all five learning algorithms.

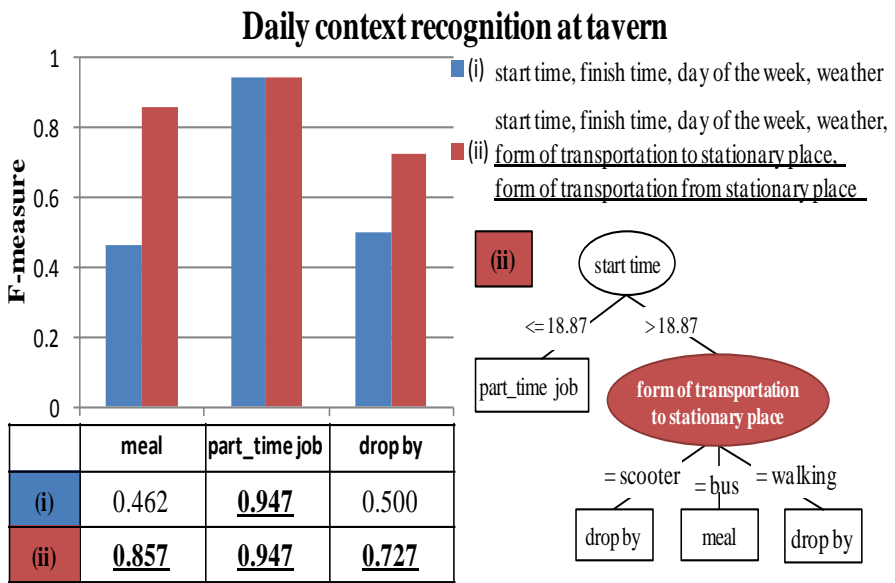

Fig. 5. Differences in accuracies of daily context recognition at a tavern by using J48 algorithm for combinations of explanatory variables, and the decision tree

TABLE III. MODEL CORRECTNESS FOR DAILY CONTEXT RECOGNITION AT TAVERN

\begin{tabular}{cccccc}
\hline \hline Model & $\begin{array}{c}\text { Random } \\
\text { Forest }\end{array}$ & J48 & SVM & $\begin{array}{c}\text { Neural } \\
\text { Network }\end{array}$ & $\begin{array}{c}\text { Bayesian } \\
\text { Network }\end{array}$ \\
\hline $\begin{array}{c}\text { Meal } \\
\text { Part-time }\end{array}$ & 0.667 & 0.857 & 0.600 & $\underline{\mathbf{0 . 9 2 3}}$ & $\underline{\mathbf{0 . 9 2 3}}$ \\
job & 0.952 & 0.947 & 0.952 & $\underline{\mathbf{1 . 0 0 0}}$ & 0.947 \\
Drop by & 0.545 & 0.727 & 0.769 & $\underline{\mathbf{0 . 9 0 9}}$ & 0.833 \\
Overall & 0.763 & 0.863 & 0.806 & $\underline{\mathbf{0 . 9 5 4}}$ & 0.910 \\
\hline
\end{tabular}

Comparative experiments were performed for two cases. One case included the start time, finish time, day of the week and weather as the explanatory variables. The second case added the form of transportation to (from) the stationary place. We confirmed in this experiment that the form of transportation is also effective as an explanatory variable. As indicated in Figure 5, it is possible to improve the levels of accuracy for "meal" and "drop by" by approximately two times and 0.23 , respectively. As shown in the decision tree, for the contexts classified as "part-time job" or any of the others at the start time, the form of transportation to the stationary place is used for the classification. This variable achieves higher levels of contextual accuracy for the "meal" and "drop by" variables. This could mean that many young people rarely drive a car or ride a scooter when they are going to have a meal at a tavern because the probability of drinking alcohol there is higher. TABLE III lists the correctness of the model generated from a training data set of 22 (meal: 6, part-time job: 10, drop by: 6). As specified in this table, although the recognition accuracy of "part-time job" is high for all of the algorithms, the overall accuracy of the random forest algorithm is the lowest, unlike in the previous evaluations. We presume that the benefits of group learning are not applicable because the training data set is too small. However, even though the training data set was small, the neural network algorithm achieved high levels of accuracy.

\section{4) Daily context recognition at a university}

GPS data were collected for one subject (one of the authors) for two months. Figure 6 shows the accuracies of the daily context recognition at a university by using the random forest algorithm. TABLE IV lists the correctness of the models generated by the five learning algorithms and the accuracies for the test data.

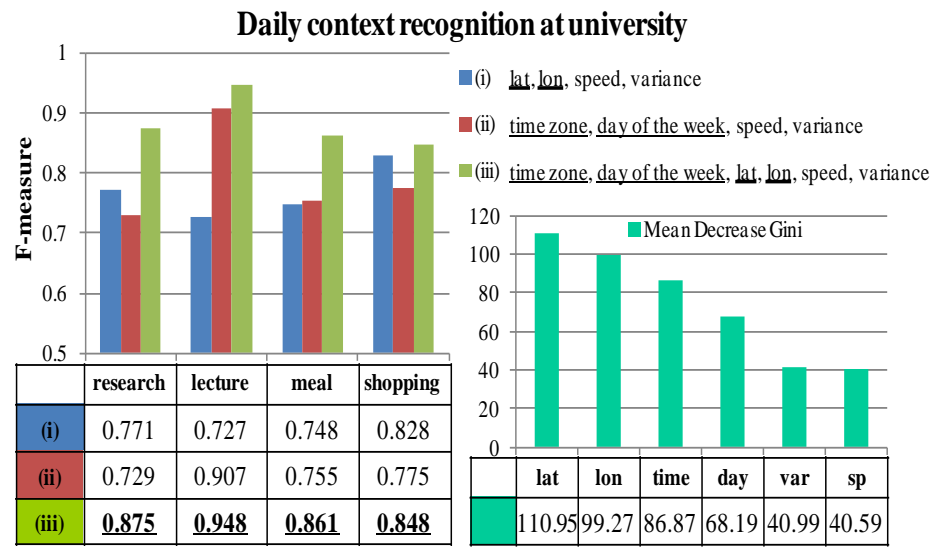

Fig. 6. Differences in accuracies of daily context recognition at a university by using random forest algorithm for combinations of explanatory variables, and the mean decrease Gini of explanatory variables

TABLE IV. MODEl CORRECTNESS AND ACCURACIES OF TEST DATA FOR DAILY CONTEXT RECOGNITION AT UNIVERSITY

\begin{tabular}{cccccc}
\hline \hline $\begin{array}{c}\text { Model / } \\
\text { Test }\end{array}$ & $\begin{array}{c}\text { Random } \\
\text { Forest }\end{array}$ & J48 & SVM & $\begin{array}{c}\text { Neural } \\
\text { Network }\end{array}$ & $\begin{array}{c}\text { Bayesian } \\
\text { Network }\end{array}$ \\
\hline \multirow{2}{*}{ Research } & $0.875 /$ & $0.831 /$ & $0.665 /$ & $0.473 /$ & $0.666 /$ \\
& $\underline{\mathbf{0 . 9 1 2}}$ & 0.861 & 0.845 & 0.639 & 0.763 \\
Lecture & $0.948 /$ & $0.921 /$ & $0.874 /$ & $0.867 /$ & $0.842 /$ \\
& $\underline{\mathbf{0 . 9 6 7}}$ & 0.961 & 0.944 & 0.898 & 0.872 \\
Meal & $0.861 /$ & $0.832 /$ & $0.762 /$ & $0.741 /$ & $0.800 /$ \\
& $\underline{\mathbf{0 . 9 1 3}}$ & 0.830 & 0.887 & 0.773 & 0.815 \\
Shopping & $0.848 /$ & $0.823 /$ & $0.729 /$ & $0.679 /$ & $0.822 /$ \\
& $\underline{\mathbf{0 . 8 8 7}}$ & 0.843 & 0.834 & 0.831 & 0.845 \\
Overall & $\underline{0.883 /}$ & $0.852 /$ & $0.757 /$ & $0.690 /$ & $0.782 /$ \\
& $\underline{\mathbf{0 . 9 2 0}}$ & 0.874 & 0.878 & 0.785 & 0.824 \\
\hline
\end{tabular}

Comparative experiments were conducted for three specific cases. Case (i) used the latitude, longitude, speed, and variance as explanatory variables. Case (ii) used the time zone, day of the week, speed, and variance. Case (iii) used all the variables. Case (iii) achieved the highest levels of accuracy. The most effective variables are latitude and longitude and the next most effective variables are time zone and day of the week. This means that the contexts at the university were determined by the time and location because many periodic activities took place there. TABLE IV lists the correctness of the model generated from a training data set of 600 (150 pieces of data per variable) and the evaluation results when using the training data set and a test data set of 300 (65 pieces of data per variable). As shown in this table, the random forest algorithm achieves the highest levels of accuracy-above approximately 0.900 - for all contexts at the university except for "shopping", which was less than 0.900 . We assume the reason for this is that "shopping" is an irregular context based on time and the 
fact that the store was located on the second floor of the same building as the cafeteria ("meal"). It is possible to improve the accuracy by using altitude values that the GPS sensor can obtain as an explanatory variable.

\section{B. Effect of Filtering}

A threshold-based filtering method was used. The GPS sensor in a mobile phone can obtain values of accuracy as an error range. It is necessary to remove the data with large positioning errors because the GPS sensor measures a lot of noise when the sensor is indoors. The noise data were removed to obtain the information discussed in this paper by using the accuracy values of the mobile phone. The purpose in this experiment was to determine a suitable threshold. Figure 7 shows the experimental results.

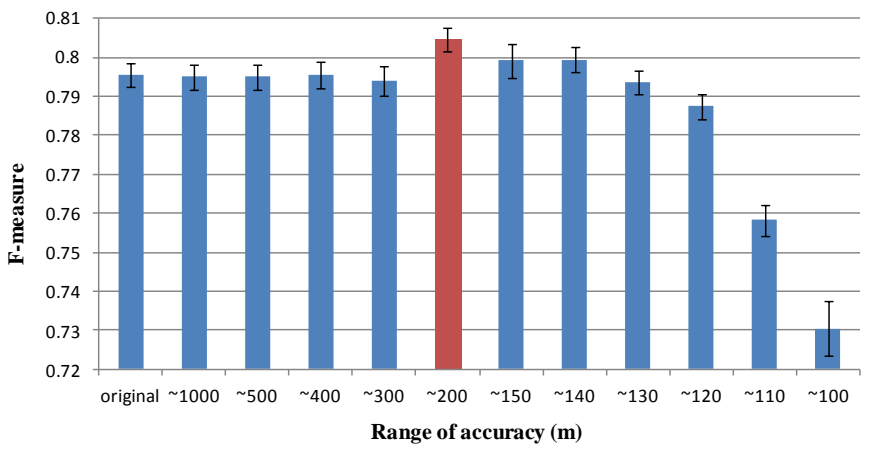

Fig. 7. F-measure when removing data by changing allowable range of accuracy

This experiment used a data set of 400 (research: 100, meeting: 100, meal: 100, shopping: 100) at the university as an original data set. The original data set contained data with a maximum accuracy of 2,373 (m) and a minimum accuracy of 7 (m). The average level of accuracy was $116.92(\mathrm{~m})$ from all the data. This result was determined on the basis of ten runs using the random forest algorithm. As shown in Figure 7, when removing the data in which the level of accuracy was higher than 200, the context recognition achieved the highest level of accuracy (0.805 overall). At this time, the number of data pieces was 385 (15 pieces were removed). When the number of data pieces was less than 323 , the recognition accuracy also began to decrease, as shown in the results for the allowable accuracy range up to 130 . The level of accuracy does not improve unless a certain minimum number of data pieces are used while removing any noise. In other words, by setting 200 as the threshold, recognition is possible with a high level of accuracy.

\section{Indoor Localization Technology}

It is possible to improve the accuracy of daily context recognition by using indoor localization technology. Thus far, other research has proposed indoor localization technologies using Wi-Fi, Japan's Indoor Messaging System (IMES), Dead Reckoning, and others [32]-[36]. Indoor localization is one of the most important elements for daily context recognition. If indoor localization technologies are added to the proposed method, it is possible to recognize more specific contexts for lifelog generations.
For indoor localization in this study, motion sensors were installed in a grid pattern on the ceiling of the authors' laboratory. An experiment was performed to recognize the contexts in the laboratory by using the motion sensor data. Figure 8 shows the experimental results and environment.

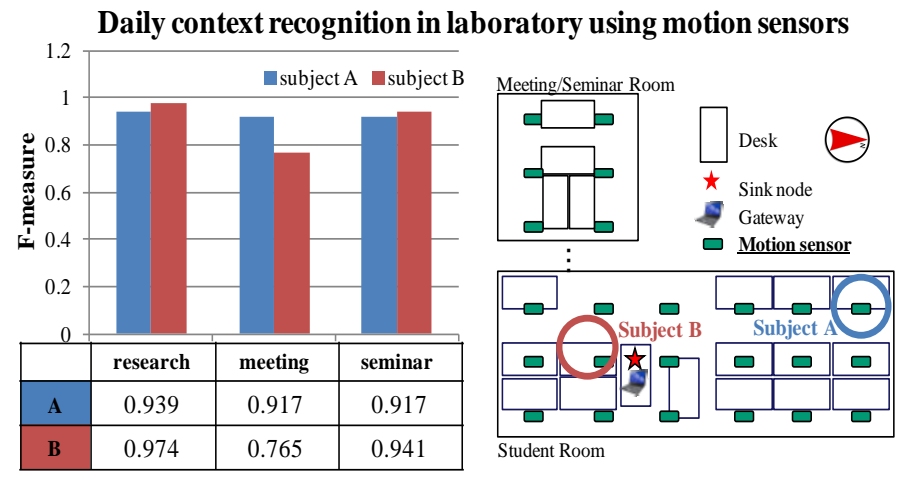

Fig. 8. Results of context recognition in laboratory by using motion sensor data in the experimental environment

Twenty-four motion sensors were installed on the ceiling of the laboratory. Six of them were installed in the meeting/seminar room and the others were installed in the student room. All the motion sensors transmitted sensing data to the gateway by detecting people movement once per minute. The experiment was performed with two participants. Participant A had the following data: 168 "research", 166 "meeting", and 118 "seminar". Participant B had the following data: 341 "research", 104 "meeting", and 20 "seminar". For both participants, the recognition accuracies were above 0.765 , and almost all the contexts had a high level of accuracy above 0.900. It is judged that the motion detection from the motion sensors showed regularity. The motion sensor just above the desk of the participants detected many movements when the participants were studying in their seats. The motion sensors installed in the meeting/seminar room detected many movement while the motion sensors installed just above the desk did not detect many movements during a group meeting in the meeting/seminar room. Moreover, when everyone was attending a seminar, the motion sensors installed in the meeting/seminar room detected many movements, and, as expected, the motion sensors installed in the student room hardly detected any movement.

\section{Healthcare Service}

The management of daily calorie consumption is considered a healthcare service in this paper. Metabolic equivalents (METS) are used to compute the energy consumed during each daily activity. METS is defined as the ratio of a person's working metabolic rate relative to the resting metabolic rate. A person's calorie consumption can be easily calculated by using the METS value as follows:

$$
E E(k c a l)=1.05 \times \operatorname{METS} \times \operatorname{Duration}(\text { hour }) \times \text { Weight }(\mathrm{kg}) .
$$

Standard tables provide the METS values for a wide range of exercises and activities. TABLE V itemizes some examples of METS values. 
TABLE V. METS EXAMPLES

\begin{tabular}{cc}
\hline \hline Examples & METS \\
\hline $\begin{array}{c}\text { driving a car, sitting - light office work (research), } \\
\text { sitting - meeting, sitting - eating }\end{array}$ & 1.5 \\
\hline eating - talking, walking - less than $2.0 \mathrm{mph}$ & 2.0 \\
\hline riding a scooter, stretching & 2.5 \\
\hline walking - 2.5 mph, weight lifting & 3.0 \\
\hline walking - 5.0 mph, carrying heavy loads & 8.0 \\
\hline
\end{tabular}

As shown in TABLE V, METS values can vary. For example, the METS values for walking depend on the speed. Since the proposed method can calculate the speed from the GPS data, it is possible to closely calculate calorie consumption.

Appropriate recommendations are needed to improve a person's lifestyle for the prevention of lifestyle-related diseases. This paper describes some of the following examples of recommendations.

$\checkmark$ If eating a meal is recognized as occurring at an irregular time or not taken three times a day, the user is advised to have a regular eating habit.

$\checkmark$ If riding a scooter is recognized for a short duration many times in a week, the user is advised to walk once in a while.

$\checkmark \quad$ If the expenditure of calories is low for a week, the user is advised to spend more time doing exercises such as stretching and playing sports.

$\checkmark \quad$ If the user is found performing research while seated in a chair for a long time, the user is advised to stand up, walk around and possibly do some light shopping.

It is possible to generate high-quality lifelogs by recognizing the specific contexts in a person's daily life. Several healthcare services can be created by using the lifelogs, enabling people to improve their lifestyles and prevent lifestyle-related diseases.

\section{E. Discussions}

With this research, we are interested in context tracking as a simple way to track people's activities, rather than describing and characterizing the taxonomy for a lifelog. Other studies have considered context estimation of daily life by using multiple sensor devices [1], but our originality is our measurement of several variables for daily context recognition by using a commercial device.

The precision of a general-purpose mobile phone for collecting information is often less than that of specialized wearable sensors. However, our estimation technique works well with mobile phone systems, and our advantage is that the mobile phone allows the collecting of daily life data in a way that is simpler than with wearable sensors. This could be an important achievement in the field of consumer electronics.

\section{CONCLUSION}

This paper described in detail the proposed method of daily context recognition for lifelog generation to prevent lifestyle- related diseases. The proposed method enables the recognition of several user contexts by using machine learning on GPS data from smartphones. We found that the optimal explanatory variables depend on the types of contexts recognized. Most contexts can be recognized by the random forest algorithm with high accuracy. The experimental results demonstrate that it is possible to improve the recognition accuracy by using threshold-based filtering and indoor localization technology. Moreover, lifelogs generated by using the proposed method can help adapt healthcare services in accordance with the user's location and context. In our future work, we will focus on adapting the proposed method for large-scale outdoor facilities by using social data as the explanatory variables. We also intend to consider a low-cost learning technique by taking a non-parametric approach to generalization. For creating context-aware services with consumer devices, we will strive for a well-balanced approach, so that individuals receiving input from these lifelogs do not find the process intrusive.

\section{REFERENCES}

[1] M. Ono, K. Nishimura, T. Tanikawa, and M. Hirose, "Neural Network Based Event Estimation on Lifelog from Various Sensors," IEEE 16th International Conference on Virtual Systems and Multimedia (VSMM), pp. 84-87, 2010.

[2] M. Abe, D. Fujioka, and H. Handa, "A Life Log Collecting System Supported by Smartphone to Model Higher-Level Human Behaviors," 2012 Sixth International Conference on Complex, Intelligent, and Software Intensive System (CISIS), pp. 665-670, 2012.

[3] D. Feldman, A. Sugaya, C. Sung, and D. Rus, "iDiary: From GPS Signals to a Text-Searchable Diary," 11th ACM Conference on Embedded Networked Sensor System (SenSys), Article No. 6, 2013.

[4] World Health Organization, WORLD HEALTH STATISTICS 2012, pp. 34-37, 2012.

[5] G. Tanaka, and H. Mineno, "A Method of Estimating Outdoor Situation for Lifelog Generation," 2nd IEEE Global Conference on Consumer Electronics (GCCE2013), pp. 361-362, 2013.

[6] O. D. Lara, and M. A. Labrador, "A Mobile Platform for Real-time Human Activity Recognition," IEEE Consumer Communications and Networking Conference (CCNC), pp. 667-671, 2012.

[7] J. B. Gomes, S. Krishnaswamy, M. M. Gaber, P. A. C. Sousa, and E. Menasalvas, "MARS: A Personalised Mobile Activity Recognition System," IEEE 13th International Conference on Mobile Data Management (MDM), pp. 316-319, 2012.

[8] J. R. Kwapisz, G. M. Weiss, and S. A. Moore, "Activity Recognition using Cell Phone Accelerometers," ACM SIGKDD Explorations Newsletter, 12(2), pp. 74-82, 2010.

[9] Y. Hattori, S. Inoue, and G. Hirakawa, "A Large Scale Gathering System for Activity Data with Mobile Sensors," 15th IEEE Annual International Symposium on Wearable Computers (ISWC), pp. 97-100, 2011.

[10] T. Maekawa, and S. Watanabe, "Unsupervised Activity Recognition with User's Physical Characteristics Data," 15th IEEE Annual International Symposium on Wearable Computers (ISWC), pp. 89-96, 2011.

[11] H. Martin, A. M. Bernardos, J. Iglesias, and J. R. Casar, "Activity logging using lightweight classification techniques in mobile devices," Personal and Ubiquitous Computing, vol. 17, no. 4, pp. 675-695, 2013.

[12] K. Cho, N. Iketani, H. Setoguchi, and M. Hattori, "Human Activity Recognizer for Mobile Devices with Multiple Sensors," Symposia and Workshop on Ubiquitous, Autonomic and Trusted Computing (UICATC), pp. 114-119, 2009.

[13] R. Liu, T. Chen, and L. Huang, "Research on Human Activity Recognition Based on Active Learning," 9th International Conference on Machine Learning and Cybernetics (ICMLC), pp. 11-14, 2010. 
[14] N. Ogawa, K. Kaji, and N. Kawaguchi, "Effects of Number of Subjects on Activity Recognition: Findings from HASC2010corpus," International Workshop on Frontiers in Activity Recognition using Pervasive Sensing (IWFA), pp. 48-51, 2011.

[15] N. Kawaguchi et al., "HASC2011corpus: Towards the Common Ground of Human Activity Recognition," 13th International Conference on Ubiquitous Computing (Ubicomp), ACM, pp. 571-572, 2011.

[16] N. Kawaguchi et al., "HASC2012corpus: Large Scale Human Activity Corpus and Its Application," 2nd International Workshop of Mobile Sensing: From Smartphones and Wearables to Big Data, pp. 10-14. 2012.

[17] A. Anjum, and M. U. Ilyas, "Activity Recognition Using Smartphone Sensors," IEEE Consumer Communications and Networking Conference (CCNC), pp. 914-919, 2013.

[18] Y-W. Bai, S-C. Wu, and C-L. Tsai, "Design and implementation of a fall monitor system by using a 3-axis accelerometer in a smartphone," IEEE Trans. Consumer Electron., vol. 58, no. 4, pp. 1269-1275, 2012.

[19] T. Suzuki, and M. Inoue, "Lifestyle Improvement Support System Considering Context of a User," IEEE International Conference on Consumer Electronics (ICCE), pp. 454-455, 2013.

[20] H. Yan, H. Huo, Y. Xu, and M. Gidlund, "Wireless sensor network based e-health system: implementation and experimental results," IEEE Trans. Consumer Electron., vol. 56, no. 4, pp. 2288-2295, 2010.

[21] A. M. Khan, and M. H. Siddiqi, "Promoting a Healthier Life-Style Using Activity-Aware Smartphones," 4th International Conference on Intelligent and Advanced Systems (ICIAS), pp. 7-11, 2012.

[22] H. O'Brien, P. van de Ven, J. Nelson, and A. Bourke, "Smartphone Interfaces to Wireless Health Sensors," 12th IEEE International Conference on e-Health Networking Applications and Services (Healthcom), pp. 180-186, 2010.

[23] I. Yamada, and G. Lopez, "Wearable Sensing Systems for Healthcare Monitoring," 2012 Symposium on VLSI Technology (VLSIT), pp. 5-10, 2012.

[24] R. Albatal, C. Gurrin, J. Zhou, Y. Yang, D. Carthy, and N. Li, "SenseSeer Mobile-Cloud-Based Lifelogging Framework," IEEE International Symposium on Technology and Society (ISTAS), pp. 144146, 2013.

[25] Z. Li, Z. Wei, W. Jia, and M. Sun, "Daily Life Event Segmentation for Lifestyle Evaluation Based on Multi-Sensor Data Recorded by a Wearable Device," 35th Annual International Conference on the IEEE
Engineering in Medicine and Biology Society (EMBC), pp. 2858-2861, 2013.

[26] Y.-S. Son, T. Pulkkinen, and J.-H. Park, "Active Monitoring for Lifestyle Disease Patient Using Data Mining of Home Sensors," IEEE International Conference on Consumer Electronics (ICCE), pp. 276-277, 2013.

[27] Z. W. Zhao, L. Liu, Q. Ma, W.-D. Li, and C.-C. Li, "A Machine-toMachine Based Framework for Diabetes Lifestyle Management," 10th IEEE International Conference on Networking, Sensing and Control (ICNSC), pp. 562-567, 2013.

[28] A. M. Khan, and S.-W. Lee, "Need for a Context-Aware Personalized Health Intervention System to Ensure Long-Term Behavior Change to Prevent Obesity," 5th International Workshop on Software Engineering in Health Care (SEHC), pp. 71-74, 2013.

[29] N. Alshurafa et al., "Designing a robust activity recognition framework for health and exergaming using wearable sensors," IEEE Journal of Biomedical and Health Informatics, DOI 10.1109/JBHI.2013.2287504, 2013.

[30] S. Suzuki et al., "Development of a topic providing system with inference of behaviors from daily life," Computer Technology and Application, vol. 4, no. 3, pp. 144-152, 2013.

[31] M. Hall, E. Frank, G. Holmes, B. Pfahringer, P. Reutemann, and I. H. Witten, "The WEKA data mining software: an update," ACM SIGKDD Explorations Newsletter, pp. 10-18, 2009.

[32] H. Liu et al., "Push the Limit of WiFi Based Localization for Smartphones," 18th Annual International Conference on Mobile Computing and Networking (Mobicom'12), pp. 305-316, 2012.

[33] K. Wu, J. Xiao, Y. Yi, M. Gao, and L. M. Ni, "FILA: Fine-grained Indoor Localization,” IEEE 2012 INFOCOM, pp. 2210-2218, 2012.

[34] Y. Sakamoto, H. Arie, T. Ebinuma, K. Fujii, and S. Sugano, "HighAccuracy IMES Localization Using a Movable Receiver Antenna and a Three-axis Attitude Sensor," International Conference on Indoor Positioning and Indoor Navigation (IPIN), pp. 1-6, 2011.

[35] Y. Sakamoto, T. Ebinuma, K. Fuji, and S. Sugano, "GPS-compatible Indoor-positioning Methods for Indoor-outdoor Seamless Robot Navigation," IEEE International Workshop on Advanced Robotics and its Social Impacts, pp. 95-100, 2012.

[36] H. Wang et al., "No Need to War-Drive: Unsupervised Indoor Localization," 10th International Conference on Mobile Systems, Applications, and Services (MobiSys'12), pp. 197-210, 2012. 